\title{
Glucocorticoid Receptor (NR3C1) Gene Polymorphism Moderate Intervention Effects on the Developmental Trajectory of African-American Adolescent Alcohol Abuse
}

\author{
Yao Zheng ${ }^{1,2}$ • Dustin Albert ${ }^{3}$ Robert J. McMahon ${ }^{1,2} \cdot$ Kenneth Dodge $^{4,5}$ • \\ Danielle Dick $^{6,7} \cdot$ the Conduct Problems Prevention Research Group
}

(C) Society for Prevention Research 2016

\begin{abstract}
Accumulative evidence from recent genotype $\times$ intervention studies suggests that individuals carrying susceptible genotypes benefit more from intervention and provides one avenue to identify subgroups that respond differentially to intervention. This study examined the moderation by glucocorticoid receptor $(N R 3 C 1)$ gene variants of intervention effects on the developmental trajectories of alcohol abuse through adolescence. Participants were randomized into Fast Track intervention and control groups self-reported past-year alcohol abuse annually from grade 7 through 2 years post-high school and provided genotype data at age 21 (69\% males; European Americans [EAs] $=270$, African-Americans [AAs] = 282). Latent growth curve models were fit to examine developmental trajectories of alcohol abuse. The interactions of 10 single nucleotide polymorphisms (SNPs) in NR3C1 with intervention were examined separately. Both EAs and AAs showed significant increases in past-year alcohol abuse with
\end{abstract}

Electronic supplementary material The online version of this article (doi:10.1007/s11121-016-0726-4) contains supplementary material, which is available to authorized users.

Yao Zheng

yao_zheng@sfu.ca

the Conduct Problems Prevention Research Group

1 Institute for the Reduction of Youth Violence, Department of Psychology, Simon Fraser University, RCB 5246, 8888 University Drive, Burnaby, BC V5A 1S6, Canada

2 BC Children's Hospital, Vancouver, BC, Canada

3 Department of Psychology, Bryn Mawr College, Bryn Mawr, PA, USA substantial inter-individual differences in rates of linear growth. AAs showed lower general levels and slower rates of linear growth than EAs. Adjusting for multiple tests, one NR3C1 SNP (rs12655166) significantly moderated intervention effects on the developmental trajectories of alcohol abuse among AAs. Intervention effects on the rates of linear growth were stronger among AAs carrying minor alleles than those not carrying minor alleles. The findings highlight the importance of taking a developmental perspective on adolescent alcohol use and have implications for future intervention design and evaluation by identifying subgroups that could disproportionally benefit from intervention.

Keywords Differential susceptibility - Glucocorticoid receptor genes - Alcohol use - Adolescent - Developmental trajectory $\cdot$ Genotype-environment interaction
4 Center for Child and Family Policy, Duke University, Durham, NC, USA

5 Department of Psychology and Neuroscience, Duke University, Durham, NC, USA

6 College Behavioral and Emotional Health Institute, Virginia Commonwealth University, Richmond, VA, USA

7 Departments of Psychology, African American Studies, and Human \& Molecular Genetics, Virginia Commonwealth University, Richmond, VA, USA 


\section{Introduction}

Alcohol and other drug use during adolescence remains a major public health issue. Adolescent alcohol use, particularly when initiated at an early age and abuse (e.g., binge drinking, drunkenness), is strongly associated with a wide variety of problems and adverse outcomes in late adolescence and young adulthood, such as driving under the influence, suicidal ideation, risky sexual behavior, substance use disorder, and antisocial behavior (Duncan et al. 1997; Newcomb and Bentler 1988). Globally, alcohol use and related problems (e.g., injury and disease) bring tremendous societal costs and economic burden (Rehm et al. 2009). Therefore, effective and early interventions with long-term effects are needed to prevent adolescent alcohol use.

The base rates of alcohol use and abuse in the general population are typically low in early adolescence, but the proportion of youth who use alcohol increases throughout adolescence (Johnston et al. 2010; SAMHSA 2002, 2013). A developmental perspective on alcohol use in adolescence emphasizes the importance of examining the growth pattern of alcohol use (e.g., increase or decline) over this developmental period using longitudinal modeling such as latent growth curve models (Duncan et al. 2006; Schulenberg and Maggs 2002). Individual differences in the rates of growth (e.g., faster or slower increase) in alcohol use and abuse are likely to be more predictive of subsequent problems and adverse outcomes. For instance, it has been shown that faster increase in alcohol use during adolescence is associated with more antisocial behavior and substance use problems in young adulthood (Duncan et al. 1997; Mason et al. 2010). In addition, large-scale national surveys have demonstrated considerable racial/ethnic differences in alcohol use and abuse during adolescence. Notably, African-American (AA) adolescents generally report lower levels of alcohol use than European American (EA) adolescents (French et al. 2002; SAMHSA 2002). However, AAs who use alcohol experience more alcohol-related problems than EAs (French et al. 2002; Wallace 1999). This finding highlights the importance of better understanding ethnic differences in developmental trajectories of alcohol use.

Meta-analyses and reviews have demonstrated the efficacy and effectiveness of many interventions preventing alcohol use in adolescents and young adults (Foxcroft et al. 2003; Smit et al. 2008; Tobler et al. 2000). Particularly, many interventions with randomized control trials (RCTs) have been shown to reduce the rates of growth of alcohol use during adolescence (e.g., Mason et al. 2003; Taylor et al. 2000). However, intervention impacts generally have modest effect size, especially with regard to long-term effects. To better allocate limited intervention resources and to achieve optimal and long-term outcomes, research has begun to prioritize the identification of subgroups of the population that respond differentially to interventions, as well as individual characteristics that identify these subgroups (Bakermans-Kranenburg and van Ijzendoorn 2015; Bloom and Michalopoulos 2013). These individual characteristics can provide potential markers or targets for future adaptive and tailored interventions to maximize the fit between individuals and intervention programs to strengthen and bolster intervention effects.

One promising avenue for detecting differential responses to interventions comes from genotype-environment interaction based on genetic differential susceptibility (Belsky et al. 2007; Belsky and Pluess 2009). Genotype-environment interaction studies suggest that genetic susceptible individuals are especially responsive to environmental influences. Under negative environmental conditions without sufficient resources and support, genetic susceptible individuals develop more adverse outcomes than less susceptible individuals; in positive conditions with ample resources and support such as intervention, however, genetic susceptible individuals achieve better outcomes (Belsky et al. 2007; Belsky and Pluess 2009). Supportive evidence in intervention research has been accumulating, including various outcomes such as externalizing behavior, substance use, and risky sexual behavior (Bakermans-Kranenburg and van Ijzendoorn 2015; van Ijzendoorn and Bakermans-Kranenburg 2015). Interventions with RCTs provide stronger tests of genotype-environment interaction than correlational studies because randomized experiments provide more statistical power, reduce measurement error, and avoid the potential problem of genotypeenvironment correlation, wherein individuals' environmental experience could be driven by genetic liability (BakermansKranenburg and van Ijzendoorn 2015; van Ijzendoorn and Bakermans-Kranenburg 2015).

Most empirical studies on genetic moderation of intervention effects have focused on genetic variants involved in the regulation of the dopaminergic (e.g., dopamine receptor D4 [DRD4]; dopamine receptor D2 [DRD2]; dopamine transporter gene $[D A T 1])$ and serotonergic (e.g., the serotonin transporter gene-linked polymorphic repeat $[5-H T T L P R]$ ) neurotransmitter systems. Meta-analysis has supported their roles as susceptibility genes (van Ijzendoorn and BakermansKranenburg 2015). To our knowledge, there are currently five empirical RCT studies that have examined genetic moderation of intervention effects on adolescent alcohol use, finding supportive evidence of genetic differences in intervention susceptibility among AAs (Beach et al. 2010; Brody et al. 2009b, 2013b, 2014) and predominantly EAs (Cleveland et al. 2015). Most of these studies focused on DRD4 (Beach et al. 2010; Brody et al. 2013b, 2014; Cleveland et al. 2015), while some also examined 5-HTTLPR (Brody et al. 2009b; Cleveland et al. 2015), $D R D 2$ and $\gamma$-aminobutyric acid (GABA) receptor coding genes (Brody et al. 2013b).

Albert et al. $(2015 \mathrm{a}, \mathrm{b})$ recently evaluated whether glucocorticoid receptor (NR3C1) gene polymorphisms moderated 
the impact of the Fast Track intervention on adult externalizing psychopathology. Specifically, they found that EAs carrying a variant of NR3C1 (rs10482672) in intervention group had the lowest risk for externalizing psychopathology including substance abuse and antisocial behavior at age 25 years, the effects of which were partly mediated by childhood externalizing psychopathology and adolescent problem behavior (Albert et al. 2015a, b). Glucocorticoid receptor proteins coded by NR3C1 genes are involved in inhibitory signaling processes of the hypothalamus-pituitary-adrenal (HPA) axis, a core biological mechanism underlying human response to environmental stress. More specifically, the hypothalamus initiates a cascade of neuroendocrine signaling that mobilizes the body's resources to resolve perceived threat, with one response component being the release of the glucocorticoid hormone cortisol, which is involved crucially in the negative feedback loop that inhibits further hormone secretion and HPA axis activity to return homeostasis (DeRijk et al. 2008). Polymorphisms in NR3Cl have been associated with glucocorticoid resistance and reduced HPA axis negative feedback (DeRijk et al. 2008), as well as a variety of behavioral phenotypes including adolescent alcohol abuse (Desrivieres et al. 2011). Evidence also suggests that individual differences in glucocorticoid signaling are associated with differential response to intervention. For instance, the evaluation of an intervention aiming to reduce disruptive behaviors reported that children with low cortisol stress reactivity to experimental challenge responded less favorably than children with high cortisol stress reactivity (van de Wiel et al. 2004).

\section{The Current Study}

A key limitation of the aforementioned studies is that they only examined differences in the levels of alcohol use at a particular time, rather than differences in the rates of growth of alcohol use through adolescence. Based on prior research, it is plausible that individuals with susceptible genotypes could show increased intervention effects on the rates of growth of adolescent alcohol use. For example, one longitudinal study reported that the rates of growth of substance use among AAs during early to mid-adolescence (11 to 14 years) were slower among carriers of the 5-HTTLPR short allele variant receiving high levels of involved-supportive parenting (Brody et al. 2009a). Using longitudinal data from the Fast Track intervention and control groups, the primary goal of this study was to examine the potential moderation by $N R 3 C 1$ variants of intervention effects on the developmental trajectories, especially the rates of growth, of adolescent alcohol abuse. We fitted a latent growth curve model to eight waves of past-year alcohol abuse self-reported annually from grade 7 through 2 years post-high school to examine the developmental trajectories of alcohol abuse. Intervention status, NR3C1 genotype, as well as their interaction, was included to predict the intercept and linear slope of the latent growth curve model. Based on prior research, we expected that participants would demonstrate substantial increase in alcohol abuse throughout adolescence and that individuals carrying susceptible NR3C1 genotypes would benefit more from the Fast Track intervention than individuals without susceptible genotypes, therefore showing slower rates of increase.

\section{Methods}

\section{The Fast Track Randomized Control Trial}

The Fast Track trial was implemented as a multi-site RCT in the early 1990 s to test whether the developmental outcomes of young children at high risk for long-term antisocial behavior could be improved through random assignment to a sustained, multi-component behavioral intervention (CPPRG 1992; 2000). Participants were selected using a multiple-gating screening procedure, resulting in enrollment of 891 children with very high levels of conduct problems at the time of school entry (see Supplementary Fig. 1 for design diagram). Written informed consent from parents and oral assent from children were obtained. Parents were paid for completing interviews, and intervention-group parents were paid for group attendance. All procedures were approved by the Institutional Review Boards of participating universities.

The first stage of the screening procedure relied on teacher reports of classroom conduct problems of 9594 kindergarteners in three cohorts (1991-1993), using the Teacher Observation of Child Adjustment-Revised Authority Acceptance Score (Werthamer-Larsson et al. 1991). Children scoring in the highest $40 \%$ within cohort and site were solicited for the second gate of screening: parent-rated home behavior problems, using a 22-item instrument based on the Child Behavior Checklist (CBCL; Achenbach 1991a). Teacher and parent scores were standardized within site and summed to yield a severity-of-risk screen score. Children were selected for the study based on this risk score, moving from highest down until desired sample sizes were reached within sites, cohorts, and conditions. Nine hundred seventy-nine children (10\% of total) were solicited to yield a sample of 891 participating children (91\% consent; intervention $n=445$; control $n=446)$. At selection, participant mean age was 6.58 years $(S D=0.48)$. Ethnicity varied $(51 \% \mathrm{AA}, 47 \% \mathrm{EA}$, $2 \%$ others), and $69 \%$ were boys. The mean externalizing problem score for the Teacher's Report Form of the CBCL (Achenbach 1991b) was 1.6 SDs above the national mean. The sample was high-risk in many ways: $58 \%$ had single parents, $29 \%$ of parents had not completed high school, and $35 \%$ of families were in the lowest socioeconomic class.

Children were selected from each of four geographic sites: Durham, NC; Nashville, TN; rural PA; and Seattle, WA. 
Elementary schools $(n=55)$ in neighborhoods with very high rates of crime and economic disadvantage were divided into paired sets (one to three sets per site) matched for demographics, and one set was randomly assigned to intervention and one to control. Children who were randomly assigned to the intervention condition received 10 consecutive years of prevention services (grades 1-10; see CPPRG 1992, 2000 for details). Elementary school programming targeted social, cognitive, emotional, and self-control deficits implicated in aggression toward peers, social rejection, academic failure, and disruptive and oppositional behavior toward authorities. Programming in middle and high school years targeted issues known to be salient at critical developmental transitions (e.g., programs in middle school addressing parental supervision and adolescent decision making relevant to alcohol, tobacco, and substance use). Published evaluations of Fast Track have shown intervention-related reductions in externalizing behavior across the elementary, high school, and young adult years (e.g., CPPRG 1992, 1999, 2000, 2011, 2015).

Elementary School Phase (Grades 1-5). During grades 1-5, intervention families were offered group intervention during a 2-h "enrichment program" that included children's social-skill "friendship groups," parent-training groups, guided parentchild interaction sessions, and paraprofessional tutoring in reading. Tutors provided three additional 30-min sessions per week in reading and peer-pairing to improve friendships with classmates. Teacher consultation and a Fast Track adaptation of the teacher-implemented PATHS curriculum (Kusche and Greenberg 1995) which addresses social-cognitive skill development were implemented universally in grade 1-5 classrooms in intervention schools (except Durham, where it was prohibited) to promote social-emotional competence. Enrichment programs were held weekly during grade 1, biweekly during grade 2 , and monthly during grades $3-5$. In addition, home visiting helped parents generalize their skill learning and address individual needs. After grade 1, criterion-referenced assessments adjusted the prescribed dosage to match need.

Middle and Early High School Phase (Grades 6-10). During grades 5 and 6 , children received a middle school transition program and four parent-youth groups on topics of adolescent development; alcohol, tobacco, and drugs; and decision-making. In grades 7 and 8, eight Youth Forums addressed vocational opportunities, life skills, and summer employment opportunities. In grades $7-10$, individualized interventions addressed parent monitoring, peer affiliation, academic achievement, and social cognition. All children received Oyserman's (1996) School-to-Jobs possible-selves intervention aimed at examining emerging identity.

Regarding intervention participation, $96 \%$ of parents and $98 \%$ of children attended at least one group session during grade 1 . Of these families, $79 \%$ of parents and $90 \%$ of children attended at least $50 \%$ of prescribed group sessions. Participation decreased modestly across years, primarily due to residential moves. In grades $7-10$, intervention continued with at least $80 \%$ of all children. High intervention fidelity was ensured by manualization, regular cross-site training, and weekly clinical supervision. Outside interventions were neither encouraged nor discouraged. The full protocol can be found at http://www.fasttrackproject.org

\section{Measures}

Past-Year Alcohol Abuse The Tobacco, Alcohol, and Drugs (grades 7-12) and Tobacco, Alcohol, and Drugs-Revised (1 and 2 years post-high school) assessment instruments (Bureau of Labor Statistics 2002) were used to measure past-year alcohol abuse. The instrument measured the number of days consuming 5+ drinks and number of days drunk in the past year. Because prevalence of alcohol abuse was low, a binary outcome was constructed where $1=$ "any past-year alcohol abuse" if participants responded any days of 5+ drinking or drunk, and $0=$ "no past-year abuse" if participants responded 0 days on both items. Technical reports and documentations are available from the Fast Track website (http://www. fasttrackproject.org/data-instruments.php). Over eight waves, 92.6\% participants provided at least one wave of data and 84.6 $\%$ provided at least three waves of data. Attrition analyses compared the severity-of-risk screen score between those who provided at least one wave of data and separately between those who provided at least three waves of data, and those without any data found no significant differences for either intervention or control groups.

Genotyping Ten $\mathrm{NR} 3 \mathrm{Cl}$ single nucleotide polymorphisms (SNPs) were selected for genotyping with the aim of optimizing the degree of genetic sequence variability accounted for by the fewest number of SNPs. There are many common DNA sequence variants within the $\mathrm{NR} 3 \mathrm{Cl}$ gene. Clusters of these variants are in linkage disequilibrium (LD; inherited together and therefore non-independent). We used a reference database (HapMap version 3; http://hapmap.ncbi.nlm.nih.gov/) to identify variants that tagged independent clusters of variants (Dick et al. 2011). The 10 SNPs identified through this tagging procedure were at least partially independent in the current samples: maximum linkage disequilibrium as measured by $R^{2}$ equal to 0.56 (EA) and 0.53 (AA) (see Supplementary Fig. 2). Genotyping of the 10 SNPs was conducted using DNA extracted from buccal cells collected at the phase 21year-old Fast Track follow-up using a cytology brush. DNA extraction was performed by Penn State University. Samples were then shipped to the Virginia Institute for Psychiatric and Behavioral Genetics for genotyping. Genotyping was conducted using commercially available primer and probe 
sequences from TaqMan Assays-on-Demand (Applied Biosystems, Foster City, CA). Duplicate genotyping produced $100 \%$ concordance rates. For samples passing quality control (genotyping success rate $>80 \%$ ), call rates for the 10 SNPs ranged from 87 to $99 \%$. All SNPs were in Hardy-Weinberg equilibrium (minimum $p>.2$ for EAs and AAs). Of 439 EAs enrolled in Fast Track, 62\% $(n=270)$ provided a DNA sample that was successfully genotyped at $N R 3 C 1$ (intervention $n=$ 129 ; control $n=141)$. Of 452 AAs, $62 \%(n=282)$ were successfully genotyped (intervention $n=143$; control $n=139$ ). For EAs and AAs separately, attrition analyses comparing the genetic subsample to the complete sample on the severity-of-risk screen score found no significant differences for either intervention or control groups. Severity-of-risk screen score did not differ between control and intervention children within the genetic subsample.

\section{Analytic Strategy}

All analyses were done in Mplus 7.2 (Muthén and Muthén 1998-2012). Latent growth curve models were fit using maximum likelihood estimator with robust standard errors and a logit link function. Missing data were handled with fullinformation maximum likelihood (FIML). For latent growth curve models with binary outcomes, a continuous latent response variable $y^{*}$ is assumed that underlies the observed categorical outcomes $y$ (i.e., alcohol abuse vs. no abuse) through a time-invariant threshold (see Supplementary Fig. 3). The mean of the intercept $\left(\eta_{0}\right)$ of the latent response variable $y^{*}$ is fixed at zero for model identification and scaling (Masyn et al. 2014). Therefore, model-estimated linear slope $\left(\eta_{1}\right)$ represents rates of linear growth through time over the latent response variable $y^{*}$, and its exponential $\left(\mathrm{e}^{\eta}{ }_{1}\right)$ represents the change of the odds of adolescents having alcohol abuse relative to no abuse for one year increase. An unconditional latent growth curve model without any covariate or predictor was first fitted using all participants to establish the most parsimonious growth model. Preliminary analyses revealed a small and significant quadratic slope, as well as a non-significant variance for the quadratic slope. Therefore, the variance for the quadratic slope was fixed at zero in all subsequent analyses. Next, ethnicity ( $1=$ "AAs," $0=$ "EAs"), intervention status ( $1=$ "intervention," $0=$ "control"), and covariates (gender, severity-of-risk screen score, age, cohort, and site) were included in the latent growth curve model to predict the intercept and linear slope.

In subsequent analyses involving NR3C1 SNPs, because genome structure differs across ethnic groups and allele frequencies vary between populations, analyses were conducted separately within EA and AA groups to avoid the potential problem of population stratification (Cardon and Palmer 2003). Frequencies of the less common alleles ranged from 5 to $44 \%$ across the 10 SNPs (see Table 2). Because individuals may carry 0,1 , or 2 copies of a given allele, two models were tested for each SNP: an additive model (SNPs were coded as 0,1 , or 2 according to the number of copies of the test allele) and a dominance model (SNPs were coded as 0 if no test allele or 1 for the presence of 1 or 2 copies of the test alleles). To test the moderation by $\mathrm{NR} 3 \mathrm{Cl}$ variants of intervention effects, a series of 10 latent growth curve models (one for each SNP) were fitted, where the same set of covariates, intervention condition, SNP genotype, and their interaction term were included to predict the intercept and linear slope. A modified Bonferroni correction was used to adjust for multiple testing involving 10 SNPs (Nyholt 2004). After accounting for the linkage structure (correlations among SNPs), two sets of SNP coding schemes (additive and dominance), and separate testing for AAs and EAs, the adjusted significance criterion was $p=.0014$ for EAs and $p=.00135$ for AAs, respectively.

\section{Results}

As is shown in Table 1, the prevalence of any past-year alcohol abuse steadily increased from grade 7 to 1 year post-high school in both AAs (7.5-25.5\%) and EAs (11.5-51.3\%), which stabilized at 2 years post-high school (25.3 and

Table 1 Prevalence of past-year alcohol abuse

\begin{tabular}{lllllllll}
\hline & $\begin{array}{l}\text { Grade } 7 \\
(\%)\end{array}$ & $\begin{array}{l}\text { Grade } 8 \\
(\%)\end{array}$ & $\begin{array}{l}\text { Grade } 9 \\
(\%)\end{array}$ & $\begin{array}{l}\text { Grade 10 } \\
(\%)\end{array}$ & $\begin{array}{l}\text { Grade 11 } \\
(\%)\end{array}$ & $\begin{array}{l}\text { Grade 12 } \\
(\%)\end{array}$ & $\begin{array}{l}\text { 1 year } \\
\text { post-high school } \\
(\%)\end{array}$ & $\begin{array}{l}\text { 2 years } \\
\text { post-high school } \\
(\%)\end{array}$ \\
\hline Overall & 9.4 & 14.2 & 18.9 & 24.1 & 26.6 & 35.6 & 38.0 & 37.8 \\
European Americans & 11.5 & 17.9 & 25.4 & 30.4 & 36.0 & 47.8 & 51.3 & 49.9 \\
African-Americans & 7.5 & 11.0 & 13.1 & 18.3 & 17.7 & 24.4 & 25.5 & 25.3 \\
Intervention & 10.8 & 14.5 & 17.9 & 23.7 & 26.1 & 34.2 & 37.7 & 35.9 \\
Control & 7.9 & 14.0 & 20.0 & 24.5 & 27.3 & 37.1 & 38.4 & 39.8 \\
\hline
\end{tabular}

Note. All participants included $(N=825)$ 
$49.9 \%$, respectively). AAs consistently showed lower prevalence of any alcohol abuse in the past year than EAs.

The unconditional latent growth curve model showed a good fit, log-likelihood (LL) $\chi^{2}=204.27, d f=237$, and $n s$. For univariate model-fitted standardized residuals, overall LL $\chi^{2}=7.21, d f=8$, and $n s$. There were a significant positive linear slope $(\mu=1.04, S E=0.13, p<.001)$ and a negative quadratic slope $(\mu=-0.09, S E=0.01, p<.001)$, suggesting a linear increase of the latent continuous response variable that represents individuals' liability of having any alcohol abuse through adolescence, which decelerated slightly post-high school. Translating the rates of linear growth into the observed binary outcome, it suggested that the odds of adolescents having any alcohol abuse relative to no abuse increased by 2.83 $\left(\mathrm{e}^{1.04}\right)$ per 1 year increase. The estimated variance of the intercept and linear slope was significant. Adolescents differed significantly from each other regarding their initial latent liability for alcohol abuse at grade $7\left(\sigma^{2}=7.79, S E=1.32\right.$, $p<.001)$ and rates of linear increase $\left(\sigma^{2}=0.35, S E=0.06\right.$, $p<.001)$. The intercept and linear slope were significantly correlated with each other, $r=-0.64, S E=0.05$, and $p<.001$. Therefore, adolescents who had lower initial liability for alcohol abuse at grade 7 tended to show higher rates of linear slope. In the conditional model, intervention status predicted neither the intercept nor linear slope $(\mathrm{Bs}=0.17$ and $-0.08, S E \mathrm{~s}=0.33$ and 0.07 , respectively, $n s)$. AAs showed a significantly lower linear slope than EAs $(B=-0.18, S E=$ $0.09, p<.05)$. Therefore, for every 1 year increase, the change of odds of AAs having any alcohol abuse relative to no abuse was $16 \%\left(1-\mathrm{e}^{-0.18}\right)$ less than that of EAs.

Controlling for covariates and adjusting for multiple tests, the main effects of NR3C1 SNPs and intervention status on the intercept and slope of alcohol abuse were not significant. However, in conditional models that included the interaction terms between SNPs and intervention condition, one SNP (rs12655166) significantly moderated the effects of intervention among AAs, but not among EAs (see Fig. 1). Results were the same for additive (see Table 2) and dominance models (see Supplementary Table 1) for this SNP. Intervention effects on the linear slope were stronger among AAs carrying $C$ alleles of the SNP than those not carrying this minor allele $(B=-0.97, S E=0.24, p<.0001$; for dominance effect, $B=-1.02, S E=0.27, p<.0001)$. Therefore, among AAs, for every 1 year increase, the intervention effect on the odds of not having any alcohol abuse relative to any abuse (i.e., preventing alcohol abuse) was 1.63 times stronger $\left(\mathrm{e}^{0.97}-1\right)$ with each additional copy of this minor allele.

\section{Discussion}

Adolescent alcohol use remains a major public health concern that brings substantial economic costs and burden to society (Duncan et al. 1997; Newcomb and Bentler 1988; Rehm et al. 2009). To maximize the impact of interventions, efforts are being made to better match subgroups of the population with optimally effective intervention programs. This study examined the development trajectories of alcohol abuse through adolescence, and whether the impact of Fast Track intervention on the developmental trajectories was moderated by $N R 3 C 1$ gene polymorphisms. Consistent with prior studies (Johnston et al. 2010; SAMHSA 2002, 2013), the proportion of youths who had alcohol abuse in the past year increased steadily through adolescence. Adolescents differed from each other in their initial liability and linear slope. This finding demonstrates the benefit of taking a developmental perspective on alcohol use in adolescence (Duncan et al. 2006; Schulenberg and Maggs 2002). Additionally, AAs consistently reported lower levels and slower linear slope than EAs, similar to prior studies (Duncan et al. 2006; French et al. 2002; SAMHSA 2002). Despite their lower levels of use, AAs who use alcohol experience more alcohol-related problems than EAs (French et al. 2002; Wallace 1999). Therefore, it is important to examine ethnic differences in the
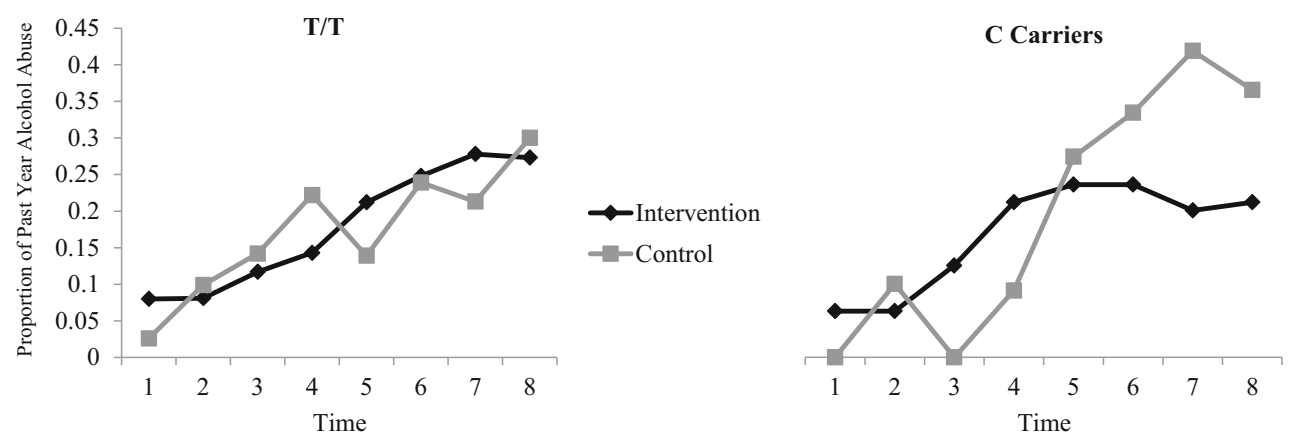

Fig. 1 Proportion of past-year alcohol abuse among African-American adolescents from grade 7 (time 1) to 2 years post-high school (time 8) by carriage of the rs 12655166 " $\mathrm{C}$ " allele and intervention status. The $T / T$ group carried no copies of the $\mathrm{C}$ allele. The $C$ Carriers group carried one

or two copies of the $\mathrm{C}$ allele. Gene-by-intervention interaction effect was statistically significant $(p<.001)$ for the linear slope of alcohol use over time 


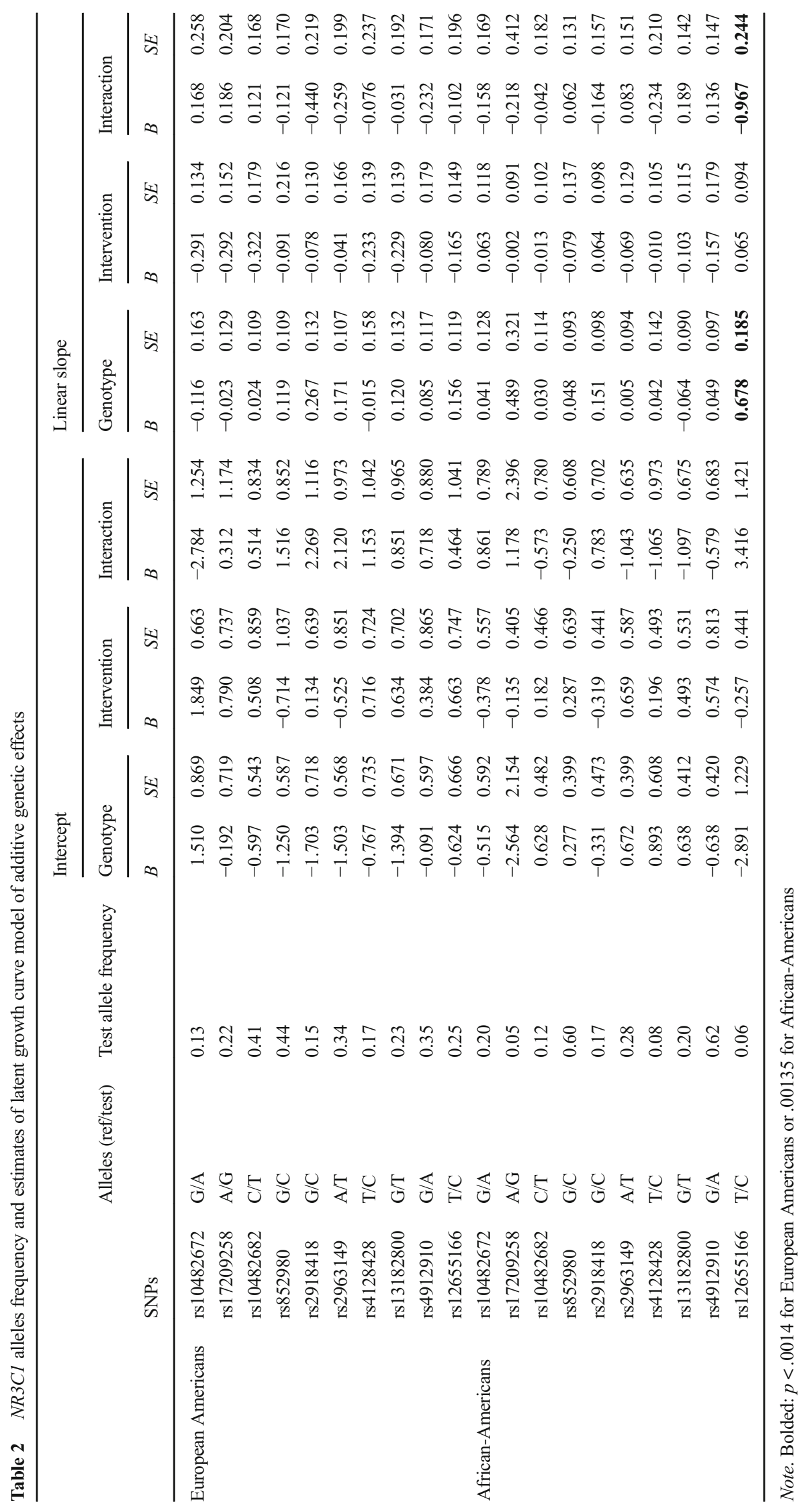


developmental trajectories of alcohol use, as well as different risk and protective factors to develop more culturally sensitive prevention programs (Metzger et al. 2013).

Current results indicated that a subgroup of the sample disproportionally benefited from the intervention. Consistent with genetic differential susceptibility (Belsky et al. 2007; Belsky and Pluess 2009), intervention effects on the linear slope were stronger among AAs carrying $\mathrm{C}$ alleles of the NR3C1 SNP rs12655166 than those not carrying this minor allele. The plausibility of this interaction is supported by consideration of the biological function of the $N R 3 C 1$ gene. Genetic sequence variation at the $N R 3 C 1$ locus is established as a source of individual differences in glucocorticoid signaling within the HPA axis, a critical mechanism involved in human response to environmental stress and challenge (DeRijk et al. 2008). When confronted with a perceived threat, the hypothalamus initiates a cascade of neuroendocrine signaling that mobilizes the body's resources toward resolving the threat. One component of this response is the release of the glucocorticoid hormone cortisol, which plays a key role in a negative feedback loop that inhibits further hormone secretion by the hypothalamus, dampens HPA axis activity, and helps to return the body to homeostasis. This inhibitory signaling is mediated by glucocorticoid receptor proteins encoded by the NR3C1 gene (DeRijk et al. 2008). Dysregulation in glucocorticoid signaling process can disrupt the response of the HPA axis to environmental stress and has been implicated in a wide array of behavioral phenotypes including child antisocial behavior (Hawes et al. 2009). Polymorphisms in NR3Cl have been associated with glucocorticoid resistance and reduced HPA axis negative feedback (DeRijk et al. 2008) and adolescent alcohol abuse (Desrivieres et al. 2011). Therefore, it is possible that the association between the alcohol abuse and the $N R 3 C 1$ polymorphism among AAs who carried the minor allele(s) that possibly led to dysregulated HPA axis function and subsequent response to environmental stress was mitigated when receiving interventions that targeted their socioemotional developmental skills and substance userelated skills and knowledge.

The NR3C1 moderation of intervention effects was only observed in AAs but not in EAs. One possible explanation relates to population stratification in genome structure and allele frequencies, which differs between ethnic groups (Cardon and Palmer 2003). The susceptibility locus identified among AAs (rs 12655166) may be a proxy for an unmeasured variant that is in high $\mathrm{LD}$ with rs 12655166 in the AAs but not in the EAs. Therefore, it is possible that we did not effectively measure and test the $N R 3 C 1$ variant responsible for differential susceptibility to intervention among EAs. Relatedly, different from Albert et al. (2015a, b) who found a SNP (rs10482672) moderated intervention effects among EAs on a static outcome (any externalizing psychopathology at age 25 years), the current study found another SNP (rs12655166) moderated intervention effects among AAs on a dynamic and different outcome (slope of alcohol abuse) in a different developmental period (i.e., adolescence). The lower levels and growth of alcohol use among AAs than for EAs, which could be attributed to differential community characteristics, peer and family processes (e.g., peer substance use, family support; Wallace 1999; Watt and Rogers 2007), could have led to the finding of genetic moderation on the slope easier than for EAs, for whom alcohol use during adolescence is regarded as more normative. Ethnic differences in these base rates and developmental processes could partly explain these differences across studies.

There are several notable strengths of this study. All previous studies that have examined genetic moderation of intervention effects on adolescent alcohol use only examined differences in the levels of use at a particular age or follow-up (typically 2-3 years later) in homogenous ethnic groups and mostly focused on early to mid-adolescence (between 11 and 15 years old), when the prevalence is generally low. This study investigated developmental trajectories in a group of ethnically diverse children followed longitudinally from grade 7 to 2 years post-high school. This design provided the opportunity to examine individual differences in the rates of growth, which have been shown to be particularly predictive of later outcomes (Duncan et al. 1997; Mason et al. 2010). A few limitations of this study warrant attention and should be addressed in future studies. First, the sample size in the genetic analyses was nevertheless small, especially as fitted to a complex model like a latent growth curve model. Replication with a larger sample size is needed for more robust conclusions. However, the experimental nature of this RCT of the Fast Track project provides more statistical power than correlational studies with equal sample size and also avoids the potential problem of gene-environment correlation (BakermansKranenburg and van Ijzendoorn 2015; van Ijzendoorn and Bakermans-Kranenburg 2015). Second, only a set of tagging SNPs that provided a parsimonious summary of all NR3C1 variation was included in the analyses, as opposed to the complete sequence of the gene. Not necessarily a limitation itself because tagging aims to capture genetic variation sufficiently without extensive genotype, genome-wide data could provide the possibility of controlling for population stratification with more advanced techniques such as genetic principal components (e.g., Cleveland et al. 2015). Third, while this study only examined one gene, $\mathrm{NR} 3 \mathrm{Cl}$, it would be more informative in future studies to investigate cumulative genetic influences using polygenic scores (e.g., Brody et al. 2013b). Fourth, the current study did not examine the mechanisms underlying the NR3C1 moderation of the intervention effect. Future studies could further investigate the extent to which genetically moderated intervention effects were mediated through putative proximal targets in the intervention (e.g., Albert et al. 2015a; Brody et al. 2014). Lastly, and most importantly, given the 
increasing concern over non-replication of candidate geneenvironment interaction research (e.g., Dick et al. 2015; Duncan and Keller 2011), extra caution should be taken when interpreting the findings. While the current study examines the theory-driven moderation of a gene selected based on its biological function with multi-year longitudinal data and rigorous measure of environment (herein intervention status), studies with much larger samples are needed to gain larger power to replicate and to more rigorously test the findings, as well as with comprehensive genotype data that could better control for population structure (e.g., Schlomer et al. 2015).

\section{Conclusion}

Current findings highlight the importance of taking a developmental perspective and examining ethnic differences in developmental trajectories of adolescent alcohol abuse. The findings also indicate the value of incorporating biological information into developmental and intervention research. Our findings suggest that individual differences in the genome lead some individuals to be more malleable than their peers to intervention effects. More specifically, our findings suggest that the integration of genetics into prevention science raises the awareness of prevention researchers that individual genetic predispositions also play a crucial role in their responsiveness to intervention and offer insights into the complex developmental mechanism underlying risk and resilience processes that could inform future intervention for refinement. Genetically informed RCTs could be incorporated into future intervention research, such as utilized in microtrials, to identify intervention components effective for individuals with different susceptibility (Brody et al. 2013a; Howe et al. 2010).

Acknowledgements This work used data from Fast Track project (for additional information concerning Fast Track, see http://www. fasttrackproject.org). We are grateful for the collaboration of the Durham Public Schools, the Metropolitan Nashville Public Schools, the Bellefonte Area Schools, the Tyrone Area Schools, the Mifflin County Schools, the Highline Public Schools, and the Seattle Public Schools. We appreciate the hard work and dedication of the many staff members who implemented the project, collected the evaluation data, and assisted with data management and analyses.

The Conduct Problems Prevention Research Group

Karen L. Bierman, Pennsylvania State University; John D. Coie, Duke University; Kenneth A. Dodge, Duke University; Mark T. Greenberg, Pennsylvania State University; John E. Lochman, University of Alabama; Robert J. McMahon, Simon Fraser University and BC Children's Hospital; and Ellen E. Pinderhughes, Tufts University.

\section{Compliance with Ethical Standards}

Funding This work was supported by National Institute of Mental Health (NIMH) Grants R18 MH48043, R18 MH50951, R18 MH50952, R18 MH50953, K05MH00797, and K05MH01027; National Institute on Drug Abuse (NIDA) Grants DA016903, K05DA15226, and P30DA023026; and Department of Education Grant
S184U30002. The Center for Substance Abuse Prevention also provided support through a memorandum of agreement with the NIMH. Additional support for the preparation of this work was provided by a LEEF B.C. Leadership Chair award, Child \& Family Research Institute Investigator Salary and Investigator Establishment Awards, and a Canada Foundation for Innovation award to Robert J. McMahon.

Conflict of Interest Drs. Bierman, Coie, Dodge, Greenberg, Lochman, McMahon, and Pinderhughes are the principal investigators on the Fast Track Project and have a publishing agreement with Guilford Publications, Inc. Royalties from that agreement will be donated to a professional organization. They are also authors of the PATHS curriculum and donate all royalties from Channing-Bete, Inc. to a professional organization. Dr. Greenberg is a developer of the PATHS curriculum and has a separate royalty agreement with Channing-Bete, Inc. Bierman, Coie, Dodge, Greenberg, Lochman, and McMahon are the developers of the Fast Track curriculum and have publishing and royalty agreements with Guilford Publications, Inc. Dr. McMahon is a coauthor of Helping the Noncompliant Child and has a royalty agreement with Guilford Publications, Inc.

Ethical Approval All procedures performed in studies involving human participants were in accordance with the ethical standards of the institutional and/or national research committee and with the 1964 Helsinki Declaration and its later amendments or comparable ethical standards.

Informed Consent Informed consent was obtained from all individual participants included in the study.

\section{References}

Achenbach, T. M. (1991a). Manual for the child behavior checklist and revised child behavior profile. Burlington: University of Vermont, Department of Psychiatry.

Achenbach, T. M. (1991b). Manual for the teacher's report form. Burlington: University of Vermont, Department of Psychiatry.

Albert, D., Belsky, D. W., Crowley, D. M., Bates, J. E., Pettit, G. S., Lansford, J. E., Dick, D., \& Dodge, K. A. (2015a). Developmental mediation of genetic variation in response to the Fast Track prevention program. Development and Psychopathology, 27, 81-95.

Albert, D., Belsky, D. W., Crowley, D. M., Latendresse, S. J., Aliev, F., Riley, B., Sun, C., Conduct Problems Prevention Research Group, Dick, D. M., \& Dodge, K. A. (2015b). Can genetics predict response to complex behavioral interventions? Evidence from a genetic analysis of the Fast Track Randomized Control Trial. Journal of Policy Analysis and Management, 34, 497-518.

Bakermans-Kranenburg, M. J., \& van Ijzendoorn, M. H. (2015). The hidden efficacy of interventions: Gene $\times$ environment experiments from a differential susceptibility perspective. Annual Review of Psychology, 66, 381-409.

Beach, S. R. H., Brody, G. H., Lei, M. K., \& Philibert, R. A. (2010). Differential susceptibility to parenting among African American youths: Testing the DRD4 hypothesis. Journal of Family Psychology, 24, 513-521.

Belsky, J., \& Pluess, M. (2009). Beyond diathesis stress: Differential susceptibility to environmental influences. Psychological Bulletin, 135, 885-908.

Belsky, J., Bakermans-Kranenburg, M. J., \& van Ijzendoorn, M. H. (2007). For better and for worse: Differential susceptibility to 
environmental influences. Current Directions in Psychological Science, 16, 300-304.

Bloom, H. S., \& Michalopoulos, C. (2013). When is the story in the subgroups? Prevention Science, 14, 179-188.

Brody, G. H., Beach, S. R., Philibert, R. A., Chen, Y. F., Lei, M. K., Murry, V. M., \& Brown, A. C. (2009a). Parenting moderates a genetic vulnerability factor in longitudinal increases in youths' substance use. Journal of Consulting and Clinical Psychology, 77, 111.

Brody, G. H., Beach, S. R., Philibert, R. A., Chen, Y. F., \& Murry, V. M. (2009b). Prevention effects moderate the association of 5-HTTLPR and youth risk behavior initiation: Gene $\times$ environment hypotheses tested via a randomized prevention design. Child Development, 80 , 645-661.

Brody, G. H., Beach, S. R., Hill, K. G., Howe, G. W., Prado, G., \& Fullerton, S. M. (2013a). Using genetically informed, randomized prevention trials to test etiological hypotheses about child and adolescent drug use and psychopathology. American Journal of Public Health, 103, S19-S24.

Brody, G. H., Chen, Y. F., \& Beach, S. R. (2013b). Differential susceptibility to prevention: GABAergic, dopaminergic, and multilocus effects. Journal of Child Psychology and Psychiatry, 54, 863-871.

Brody, G. H., Chen, Y. F., Beach, S. R., Kogan, S. M., Yu, T., DiClemente, R. J., Wingood, G. M., Windle, M., \& Philibert, R. A. (2014). Differential sensitivity to prevention programming: A dopaminergic polymorphism-enhanced prevention effect on protective parenting and adolescent substance use. Health Psychology, 33, 182-191.

Bureau of Labor Statistics. (2002). National Longitudinal Survey of Youth 1997 Cohort, 1997-2001. Chicago/Columbus, OH: University of Chicago, National Opinion Research Center/Ohio State University, Center for Human Resource Research.

Cardon, L. R., \& Palmer, L. J. (2003). Population stratification and spurious allelic association. Lancet, 361, 598-604.

Cleveland, H. H., Schlomer, G. L., Vandenbergh, D. J., Feinberg, M., Greenberg, M., Spoth, R., Redmond, C., Shriver, M. D., Zaidi, A. A., \& Hair, K. L. (2015). The conditioning of intervention effects on early adolescent alcohol use by maternal involvement and dopamine receptor D4 (DRD4) and serotonin transporter linked polymorphic region (5-HTTLPR) genetic variants. Development and Psychopathology, 27, 51-67.

Conduct Problems Prevention Research Group. (1992). A developmental and clinical model for the prevention of conduct disorder: The FAST Track Program. Development and Psychopathology, 4, 509-527.

Conduct Problems Prevention Research Group. (1999). Initial impact of the Fast Track prevention trial for conduct problems: I. The high-risk sample. Journal of Consulting and Clinical Psychology, 67, 631647

Conduct Problems Prevention Research Group. (2000). Merging universal and indicated prevention programs: The Fast Track model. Addictive Behaviors, 25, 913-927.

Conduct Problems Prevention Research Group. (2011). The effects of the Fast Track preventive intervention on the development of conduct disorder across childhood. Child Development, 82, 331-345.

Conduct Problems Prevention Research Group. (2015). Impact of early intervention on psychopathology, crime, and well-being at age 25 . American Journal of Psychiatry, 172, 59-70.

DeRijk, R. H., van Leeuwen, N., Klok, M. D., \& Zitman, F. G. (2008). Corticosteroid receptor-gene variants: Modulators of the stressresponse and implications for mental health. European Journal of Pharmacology, 585, 492-501.

Desrivieres, S., Lourdusamy, A., Muller, C., Ducci, F., Wong, C. P., Kaakinen, M., Pouta, A., Hartikainen, A. L., Isohanni, M., Charoen, P., Peltonen, L., Freimer, N., Elliott, P., Jarvelin, M. R., \& Schumann, G. (2011). Glucocorticoid receptor (NR3C1) gene polymorphisms and onset of alcohol abuse in adolescents. Addiction Biology, 16, 510-513.

Dick, D. M., Latendresse, S. J., \& Riley, B. (2011). Incorporating genetics into your studies: A guide for social scientists. Frontiers in Psychiatry, 2, 1-11.

Dick, D. M., Agrawal, A., Keller, M. C., Adkins, A., Aliev, F., Monroe, S., Hewitt, J. K., Kendler, K. S., \& Sher, K. J. (2015). Candidate gene-environment interaction research: Reflections and recommendations. Perspectives on Psychological Science, 10, 37-59.

Duncan, L. E., \& Keller, M. C. (2011). A critical review of the first 10 years of candidate gene-by-environment interaction research in psychiatry. American Journal of Psychiatry, 168, 1041-1049.

Duncan, S. C., Alpert, A., Duncan, T. E., \& Hops, H. (1997). Adolescent alcohol use development and young adult outcomes. Drug and Alcohol Dependence, 49, 39-48.

Duncan, S. C., Duncan, T. E., \& Strycker, L. A. (2006). Alcohol use from ages 9 to 16: A cohort-sequential latent growth model. Drug and Alcohol Dependence, 81, 71-81.

Foxcroft, D. R., Ireland, D., Lister-Sharp, D. J., Lowe, G., \& Breen, R. (2003). Longer-term primary prevention for alcohol misuse in young people: A systematic review. Addiction, 98, 397-411.

French, K., Finkbiner, R., \& Duhamel, L. (2002). Patterns of substance use among minority youth and adults in the United States: An overview and synthesis of national survey findings (National Evaluation Data Services [NEDS] Technical Report, Center for Substance Abuse Treatment, Substance Abuse and Mental Health Services Administration [SAMHSA], contract no. 270-97-7016). Fairfax, VA: Caliber Associates.

Gabriel, S. B., Schaffner, S. F., Nguyen, H., Moore, J. M., Roy, J., Blumenstiel, B., Higgins, J., DeFelice, M., Lochner, A., Faggart, M., Liu-Cordero, S. N., Rotimi, C., Adeyemo, A., Cooper, R., Ward, R., Lander, E. S., Daly, M. J., \& Altshuler, D. (2002). The structure of haplotype blocks in the human genome. Science, 296, 2225-2229.

Hawes, D. J., Brennan, J., \& Dadds, M. R. (2009). Cortisol, callousunemotional traits, and pathways to antisocial behavior. Current Opinion in Psychiatry, 22, 357-362.

Howe, G. W., Beach, S. R., \& Brody, G. H. (2010). Microtrial methods for translating gene-environment dynamics into preventive interventions. Prevention Science, 11, 343-354.

Johnston, L. D., O'Malley, P. M., Bachman, J. G., \& Schulenberg, J. E. (2010). Monitoring the future: National survey results on drug use, 1975-2009. Volume I: Secondary School Students (NIH Publication No. 10-7584). Bethesda, MD: National Institute on Drug Abuse.

Kusche, C. A., \& Greenberg, M. T. (1995). The PATHS curriculum. Seattle: Developmental Research and Programs.

Mason, W. A., Kosterman, R., Hawkins, J. D., Haggerty, K. P., \& Spoth, R. L. (2003). Reducing adolescents' growth in substance use and delinquency: Randomized trial effects of a parent-training prevention intervention. Prevention Science, 4, 203-212.

Mason, W. A., Hitch, J. E., Kosterman, R., McCarty, C. A., Herrenkohl, T. I., \& Hawkins, J. D. (2010). Growth in adolescent delinquency and alcohol use in relation to young adult crime, alcohol use disorders, and risky sex: A comparison of youth from low- versus middle-income backgrounds. Journal of Child Psychology and Psychiatry, 51, 1377-1385.

Masyn, K. E., Petras, H., \& Liu, W. (2014). Growth curve models with categorical outcomes. In Encyclopedia of criminology and criminal justice (pp. 2013-2025). New York: Springer.

Metzger, I., Cooper, S. M., Zarrett, N., \& Flory, K. (2013). Culturally sensitive risk behavior prevention programs for African American adolescents: A systematic analysis. Clinical Child and Family Psychology Review, 16, 187-212.

Muthén, L. K., \& Muthén, B. O. (1998-2012). Mplus user's guide (7th ed.). Los Angeles, CA: Authors. 
Newcomb, M. D., \& Bentler, P. M. (1988). Consequences of adolescent drug use: Impact on the lives of young adults. Thousand Oaks: Sage Publications.

Nyholt, D. R. (2004). A simple correction for multiple testing for singlenucleotide polymorphisms in linkage disequilibrium with each other. American Journal of Human Genetics, 74, 765-769.

Oyserman, D., Sanchez-Burks, J., \& Harrison, K. (1996). Social identity and possible selves in adolescence. Unpublished manuscript, University of Michigan.

Rehm, J., Mathers, C., Popova, S., Thavorncharoensap, M., Teerawattananon, Y., \& Patra, J. (2009). Global burden of disease and injury and economic cost attributable to alcohol use and alcoholuse disorders. Lancet, 373, 2223-2233.

SAMHSA. (2002). Alcohol and cigarette use by racelethnicity and age in the NHSDA Survey (NEDS fact sheet 136). Rockville, MD: Center for Substance Abuse Treatment.

SAMHSA. (2013). Results from the 2012 National Survey on Drug Use and Health: Summary of national findings (NSDUH Series H-46, HHS Publication No. (SMA) 13-4795). Rockville, MD: SAMHSA.

Schlomer, G. L., Cleveland, H. H., Vandenbergh, D. J., Fosco, G. M., \& Feinberg, M. E. (2015). Looking forward in candidate gene research: Concerns and suggestions. Journal of Marriage and Family, 77, 351-354.

Schulenberg, J. E., \& Maggs, J. L. (2002). A developmental perspective on alcohol use and heavy drinking during adolescence and the transition to young adulthood. Journal of Studies on Alcohol, S14, 54 70.

Smit, E., Verdurmen, J., Monshouwer, K., \& Smit, F. (2008). Family interventions and their effect on adolescent alcohol use in general populations: A meta-analysis of randomized controlled trials. Drug and Alcohol Dependence, 97, 195-206.

Taylor, B. J., Graham, J. W., Cumsille, P., \& Hansen, W. B. (2000). Modeling prevention program effects on growth in substance use: Analysis of five years of data from the Adolescent Alcohol Prevention Trial. Prevention Science, 4, 183-197.

Tobler, N. S., Roona, M. R., Ochshorn, P., Marshall, D. G., Streke, A. V., \& Stackpole, K. M. (2000). School-based adolescent drug prevention programs: 1998 meta-analysis. Journal of Primary Prevention, $20,275-336$

van de Wiel, N. M., van Goozen, S. H., Matthys, W., Snoek, H., \& van Engeland, H. (2004). Cortisol and treatment effect in children with disruptive behavior disorders: A preliminary study. Journal of the American Academy of Child \& Adolescent Psychiatry, 43, 10111018.

van Ijzendoorn, M. H., \& Bakermans-Kranenburg, M. J. (2015). Genetic differential susceptibility on trial: Meta-analytic support from randomized controlled experiments. Development and Psychopathology, 27, 151-162.

Wallace, J. M. (1999). Explaining race differences in adolescent and young adult drug use: The role of racialized social systems. Drugs \& Society, 14, 21-36.

Watt, T. T., \& Rogers, J. M. (2007). Factors contributing to differences in substance use among Black and White adolescents. Youth \& Society, $39,54-74$

Werthamer-Larsson, L., Kellam, S., \& Wheeler, L. (1991). Effect of firstgrade classroom environment on shy behavior, aggressive behavior, and concentration problems. American Journal of Community Psychology, 19, 585-602. 